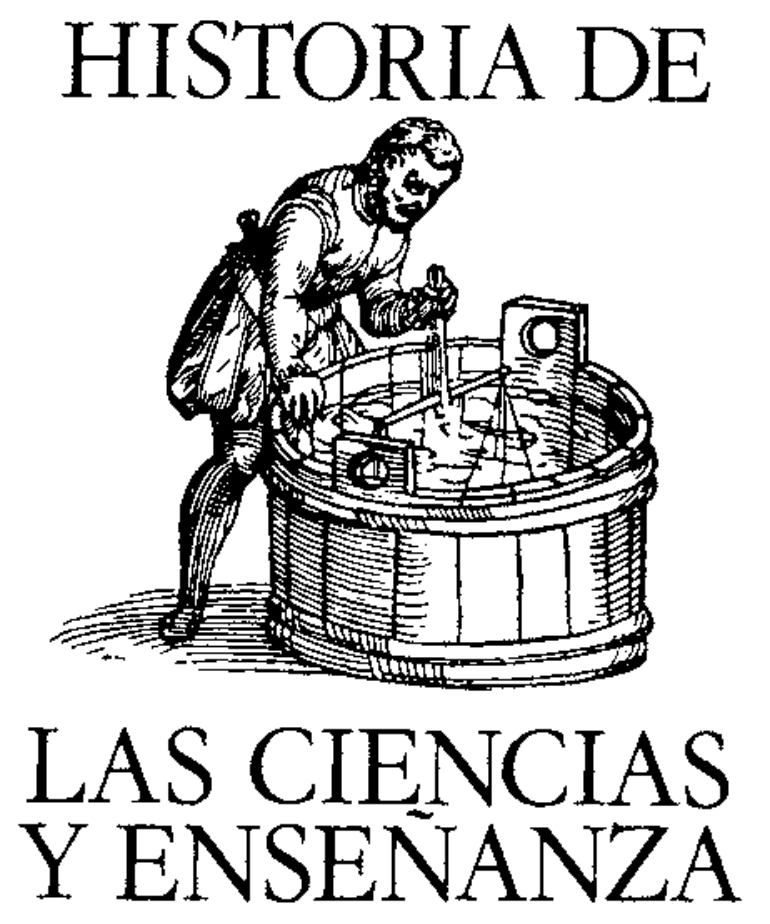

\title{
INTUICION E HISTORIA DE LAS CIENCIAS EN LA ENSEÑANZA (*)
}

VALERA, M.(1), LOPEZ FERNANDEZ, C.(2), GARCIA GARCIA, S.(3),

GIL IBAÑEZ, J.(4), FRUTOS, J.(5), INIESTA, M² A.(6), MARSET, P.(7).

(1) Dr. en Físicas, Departamento Historia de la Medicina, Murcia.

(2) Dr. en Físicas, Prof. Agregado Matemáticas, I.N.B. Alfonso X. Murcia.

(3) Ldo. en Geografía e Historia, Prof. de E.G.B. en Col. Nacional La Paz, EI Paimar (Murcia).

(4) Ldo. en Químicas, Prof. Colegio Cooperativa SANJE. Alcantarilla (Murcia).

(5) Ldo. en Físicas, Prof. Colegio Cooperativa SANJE, Alcantarilta (Murcia).

(6) Lda. en Quimicas, Prof. Agregado de Fisica y Quimica, I.N.B., Torrevieja (Alicante).

(7) Catedrático Historia de la Medicina, Dpto. Historia de la Medicina, Murcia.

${ }^{(}{ }^{*}$ Trabajo realizado en el Seminario de Historia y Enseñanza de las Ciencias del ICE de la Lniversidad de Murcia.

\section{SUMMARY}

This paper establishes a relationship between some of the students' misconceptions (in mathematics, astronomy, mechanics,...) and the historical evolution of scientific knowledge.

The results suggest that the awareness on the teacher's side of such historical evolution would help to introduce the physical concepts in a more appropiate manner.

\section{NNTRODUCCION}

Existe un tipo de conocimiento en el estudiante, que podemos calificar de intuitivo, basado en la observación directa de los fenómenos naturales y su racionalización, a través del cual va conceptua* 
lizando diversos fenómenos que observa cotidianamente. Esta conceptualización intuitiva persiste, a nuestro juicio, a pesar del conocimiento obtenido mediante la enseñanza habitualmente impartida en los centros escolares, y dicha conceptualización intuitiva tiende a reproducir las etapas del desarrollo histórico del conocimiento científico, al menos en las primeras etapas del aprendizaje (Piaget 1978) (Piaget 1969) (Piaget 1979).

Partiendo de éstas premisas iniciales, el Seminario de Historia y Enseñanza de las Ciencias de la Región Murciana elaboró y contrastó durante el curso 1981-82 unas pruebas a partir de las cuales pudieran o no corroborarse nuestras hipótesis de partida y que sirvieran, a su vez, de base para abordar en el futuro la realización de unas programaciones didácticas tendentes a superar estos inconvenientes apuntados. Pensamos, además, que a partir del conocimiento por parte del profesorado del desarrollo histórico de las Ciencias, sería posible introducir más adecuadamente los conceptos científicos correspondientes a las distintas disciplinas, sin que por ello defendamos ninguna postura exclusivista, abogando más bien porque, simultáneamente a la introducción de la Historia de las Ciencias como instrumento didáctico, se recurra a otras técnicas como la experimentación, la participación activa y otras (Navarro 1980) (Brush y King 1972). Una advertencia previa que creemos conveniente señalar es la de que todos los componentes del grupo tenemos conciencia plena de las limitaciones del trabajo actualmente realizado, por lo que las conclusiones que de él se derivan no pasan de ser consideradas por nuestra parte como provisionales, a la espera de poder corroborarlas más firmemente en pruebas posteriores. Con todo, nos parecen lo suficientemente significativas para poder ser ya adelantadas.

\section{METODOLOGIA}

La prueba base de nuestro trabajo consistió en la realización de unas encuestas que recogían una serie de conceptos básicos referentes a tres disciplinas concretas de alta significación, tanto a nivel histórico-científico como a nivel epistemológico: Mecánica, Matemáticas y Astronomía. En todo momento se ha intentado captar el grado de asimilación que los estudiantes tenian en torno a dichos conceptos así como el nivel de corrección que habían adquirido en el manejo de los mismos.

Al objeto de contrastar adecuadamente la posible persistencia del conocimiento intuitivo a través del tiempo y su posible modificación a partir de la asimilación del conocimiento adquirido académica. mente, pasamos la prueba en diversos niveles de enseñanza, $3^{\circ}, 5^{\circ}$ y $7^{\circ}$ de E.G.B., $2^{\circ}$ de F.P., $1^{\circ}$ y $2^{\circ}$ de B.U.P. El amplio rango de edades correspondiente a los mismos nos indujo a elaborar dos tipos de encuesta, uno para los cursos $3^{\circ}$ y $5^{\circ}$ de E.G.B. y otro para los restantes. Convencionalmente les denominamos, respectivamente. encuestas de primera y de segunda etapas.

Dentro de las tres áreas generales a que antes hacíamos referencia, centramos nuestra atención en una serie de cuestiones puntuales que, en la medida de lo posible, intentamos quedaran recogidas en ambas encuestas. La explicitación de estas cuestiones la realizaremos al comentar los contenidos de las encuestas (véanse Anexos I y II), refiriéndonos por separado a cada una de las áreas temáticas.

a) Matemáticas. Es sabido la enorme dificultad que supuso para el desarrollo del Algebra y del Análisis Matemático el no disponer durante sigios de un sistema de numeración adecuado. Cuando por fin éste se consigue, su enorme eficacia es achacable a dos cuestiones principaimente: la introducción del cero y la utilización del principio de valor relativo o posicional. La primera pregunta en ambas encuestas apunta en esa dobie dirección, intentando poner de manifiesto cuántos alumnos tienden a introducir un simbolo especifico para el cero y cuántos manejan el principio del valor relativo al escribir con simbolos diferentes a los usuales los quince primeros números, circunstancias ambas que deberían darse en concreto al escribir desde el "diez" al "quince".

La pregunta 2 de la encuesta de segunda etapa, que no tiene paralelismo en la de primera, intenta clarificar si los alumnos tienden a reproducir el obstáculo con el que tropezaron los pitagóricos: la insuficiencia de los números racionales para dar plena solución al problema de la comparación de longitudes. Presumiblemente, ello lo manifestarán respondiendo "si» al primer apartado y «no» al segundo, poniendo así de relieve que para ellos el número irracional está desconectado de toda cuestión en la que intervengan longitudes "reales». En esta misma linea general, aunque bajo una perspectiva distinta, incide la pregunta 3 , que mostraría si para los alumnos todo lo que exista a nivel teórico-formal (como los número imaginarios) ha de tener una existencia real.

Las preguntas 4, 5 y 9 de la encuesta de segunda 
etapa, que se corresponden con la 2 de la primera etapa, se plantearon con el objetivo de ver si los alumnos tienen clara la noción de kinfinito* a nivel conceptual y operativo o si, por el contrario, y como sucedió históricamente (recuérdense las paradojas de Zenón), tienden a manipular los infinitos con las mismas reglas aplicables formalmente a los finitos.

Con las preguntas 6,7 y 8 de la segunda etapa, $y$, paralelamente, con la 3 de la primera, intentamos ver si, independientemente de la posible asimilación del concepto de función a nivel académico (pregunta 6), se tiende o no a reproducir la genésis histórica del concepto de función, considerando que sólo existe relación funcional entre dos conjuntos de datos numéricos cuando entre ambos se da un nexo de relación física causal.

b) Mecánica. Los conceptos de velocidad y aceleración, así como su relación con las magnitudes básicas (espacio y tiempo), han contituído la piedra angular de la Mecánica desde que Galileo estableciera en el siglo XVII los fundamentos de la misma tras costosas investigaciones. Sin embargo, en los textos usualmente manejados en prácticamente todos los niveles de enseñanza en nuestro país, dichos conceptos son tratados muy escuetamente, dando por supuesto que su asimilación ha de ser necesariamente directa y sin dificultad por parte del alumno. Por nuestra parte, y valga como aval el hecho de que duró más de 2.000 años la génesis histórica de estos conceptos, mantenemos la idea contraria, pensando que el tratar tan superficialmente ideas tan básicas y fundamentales no lleva sino a una negativa asimilación de las mismas.

Las preguntas 10,11 y 13 de la encuesta de segunda etapa, en concomitancia con las 4,5 y 6 de la primera, iban dirigidas a ratificar o desechar esta cuestión. Mediante ellas se fuerza a los alumnos a manejar todos los conceptos antes aludidos bajo unos enunciados que se apartan un tanto de los "académicos" que usualmente encuentran en los exámenes. Incluso la 18 podria aportar datos en este terreno.

Pasando ya a otra cuestión, es sabido que una de las contraargumentaciones más utilizadas contra la teoría heliocéntrica fue el que la Tierra dejaria atrás sobre su vertical primitiva a los cuerpos que cayeran libremente sobre ella, dificultad que Galileo salvó con la idea de la composición de movimientos. Esto realza la cuestión de intentar averiguar si dicha idea del movimiento compuesto es o no intuitivamente aceptada por nuestros alumnos. La pregunta 12 es claramente útil para ello.

Otra de las ideas que históricamente ha costado más trabajo erradicar ha sido la defendida por Aristóteles en tomo a la necesidad de que sobre todo cuerpo en movimiento actuará permanentemente un motor. Hasta qué punto ésta idea persiste en nuestros alumnos es algo sobre lo que puede aportarnos datos interesantes las preguntas 14 y 15 de la encuesta de segunda etapa.

En otro orden de cosas, la pregunta 16 de esta misma encuesta, equiparable a la 7 de primera etapa, intentaba dilucidar si el concepto de fuerza se une a nivel intuitivo con el de velocidad en lugar de con el de aceleración.

La dificultad que ha encontrado el hombre para llegar a aceptar la idea de vacio, y la posibilidad de que el mismo sea marco de fenómenos físicos, quedó históricamente bien reflejada en la famosa teoria del "horror vacui» que tantos siglos estuvo en vigor. Las preguntas 17,18 y 19 de la encuesta de segunda etapa, tienden a ver el estado de esta cuestión en nuestros estudiantes.

En esta misma encuesta, las preguntas 20,21 y 23 , en correspondencia con las 8 y 9 de la primera etapa, nos sirven para dilucidar si el concepto de peso es considerado por los alumnos como inmerso en otro más general, el de gravedad, o bien si, análogamente a lo ocurrido durante siglos, am. bos lo consideran deslindados. Asimismo, la primera de estas tres preguntas nos permite medir si el concepto de gravedad ha sido asimilado sólidamente.

c) Astronomía. Partiendo de la conocida idea defendida por Aristóteles sobre la existencia de dos mundos físicos contrapuestos, el sublunar (lleno de imperfecciones) y el supralunar (perfectamente ordenado), intentamos averiguar con las preguntas 24 y 25 de la segunda etapa y la 12 de la primera (incluso la 13), si dicha idea persiste a nivel intuitivo en nuestros alumnos, si bien, y dado el notable volumen de información que hoy día reciben los mismos sobre el espacio interestelar a través de los medios de comunicación, hemos matizado dos regiones en el mundo supralunar, correspondientes, respectivamente, al sistema solar y al exterior del mismo.

Sobre la cuestión anterior abundan, aunque bajo una perspectiva menos directa, las preguntas 26 , 27 y 28 de la encuesta de segunda etapa, en tanto 
que podrian mostrar la persistencia de la idea referida a la existencia de una esfera de las estrellas fijas, pero básicamente estas tres preguntas, junto con la 22 y la 30 , así como las $10,11,14$ y 15 de primera etapa, tendían a informamos sobre el nivel general de conocimientos astronómicos de nuestros alumnos a nivel intuitivo, pues desgraciadamente la ciencia que desencadenó la revolución científica, ha sido casi erradicada de los planes de estudio.

Fuera ya de la significación subyacente a las preguntas de las encuestas, no podemos dar por terminada la exposición de nuestra metodología sin aportar algunos datos técnicos referentes a la realización de la prueba-encuesta, objeto central de este trabajo de investigación. Así, es procedente indicar que la encuesta fue pasada en cuatro cursos, normalmente de distinto centro, por cada nivel de los cuatro señalados al principio. Por to que respecta al número de alumnos fue de 123 en $3^{\circ}$ EGB, 130 en $5^{\circ} \mathrm{EGB}, 118$ en $7^{\circ} \mathrm{EGB}, 94$ en FP 1 y 121 en $2^{\circ}$ BUP, ascendiendo en total a 586 .

Tuvimos acceso a los niveles de $3^{\circ}, 5^{\circ}$ y $7^{\circ}$ de EGB en los colegios públicos de La Alberca y $\mathrm{El}$ Palmar, en la Cooperativa SANJE y en el también colegio público de Los Barreros (Cartagena). Las pruebas para FP fueron pasadas en la Cooperativa SANJE, en los Institutos Politécnicos de Murcia y de Molina. Las correspondientes a BUP fueron llevadas a cabo en los Institutos de Bachillerato de Torrevieja (Alicante), Alfonso X (Murcia), Molina de Segura (Murcia) e Isaac Peral (Cartagena).

Previamente al pase de estas encuestas se realizó un ensayo con unas encuestas piloto pasadas en otros centros o en otros cursos distintos a los anteriores, las cuales sirvieron para una mayor aquilatación de las preguntas tanto cuantitativamente como cualitativamente. Así, el tiempo previsto inicialmente como necesario (una hora y media) fue reducido a una hora, pasando el número de preguntas de 40 a 30 para la segunda etapa y de 20 a 15 para la primera.

La acogida general de la prueba entre los alumnos fue bastante buena, aprenciándose en los mismos una total ausencia de tensión al realizarla, no tomándola como un exámen. Las encuestas no contestadas con seriedad carecen de significación dado su reducidísimo número.

\section{RESULTADOS}

Una vez tabulados adecuadamente los resultados de las encuestas y tras una amplia reflexión colectiva sobre los datos conseguidos, hemos obtenido, aunque con las reservas que ya señaábamos al principio, una buena serie de conclusiones que exponemos a continuación.

1. Matemáticas. Los resultados obtenidos para la cuestión primera de ambas encuestas («Representa los quince primeros números por simbolos distintos a los que manejas habitualmente. Por ejemplo, $5=\square, 6=\mathbb{}, 7=\bigotimes$ etc), ponen clarísimamente de manifiesto (véase tabla I) que la tendencia a utilizar el cero y el principio del valor relativo es verdaderamente irrelevante en todos los niveles, no superándose nunca el $5 \%$ para el primero y alcanzando el segundo un valor significativo tan solo en BUP, aunque el mismo no deje de ser reducido (apenas un $15 \%$ ).

Queda entonces clara la tendencia a reproducir uno de los principales "errores históricos" de la Matemática, y ello a pesar de la introducción de los sistemas de numeración en los primeros niveles de EGB, aspecto que se manifiesta como superfluo ante la evidenciación de que los alumnos parecen asimilar exclusivamente los automatismos de cálculo, quedando marginado el concepto de número.

Las cuestiones 4 y 9 de segunda etapa ("¿Qué hay más, números naturales o números impares?; ¿Podría suceder que la suma de infinitos numeros (del tipo que sean) no dé como resultado infinito?») y la 2 de la primera ( $\mathrm{Si}$ en una caja tuvieses todos los números y en otra todos los números que empiezan por uno, ¿Cual de las dos cajas crees que tendría más números?»), parecen indicar (Tabla II) que en los dos primeros niveles estudiados no se tiene noción del "infinito", tomándose a partir de ahí bruscamente conciencia del mismo (los porcentajes saltan de un $6 \%$ a más de un $40 \%$ ), si bien en $7^{\circ}$ de EGB parece haber algunas dificultades con relación a los niveles posteriores en lo que respecta a la manipulación de los "infinitos" (la diferencia porcentual de contestaciones correctas es de 15 puntos); posiblemente ello se deba a que en dicho curso aún no se ha introducido las progresiones geométricas ilimitadas. En cualquier caso, con los porcentajes obtenidos (siempre claramente inferiores al $50 \%$ ) parece evidente que las dificultades observadas históricamente hasta conseguir dar un tratamiento adecuado a los infinitos, presentes desde las paradojas de Zenón, tienden a reproducirse en nuestros alumnos. Los resuitados que obtuvimos para la pregunta 5 apuntaron en el mismo sentido, aunque al ser sus valores sustancialmente 
inferiores, dicha cuestión no resultó discriminatoria.

Los porcentajes de contestación correctos a la segunda cuestión de la encuesta de segunda etapa ("Sabiendo que $\sqrt{3}=1,7320 \ldots$ (infinitas cifras decimales) y que $3 / 4=0.75$, a) ¿Podría construirse una varilla que midiese exactamente $3 / 4$ centrimetros? b) ¿Y una que midiese exactamente $\sqrt{3 \text { cen- }}$ trímetros?") ponen de manifiesto (Tabla III) la persistencia en nuestros alumnos de otra idea históricamente controvertida en la evolución de la Matemática: la suficiencia del número racional para solucionar el problema de la medida y la no existencia real de cantidades expresadas mediante números irracionales. El porcentaje de respuestas corectas correspondiente a $7^{\circ}$ de EGB es posible que esté distorsionado por un notable grado de aleatoriedad en la contestación, dado que en este curso los estudiantes todavía no han estudiado el número irracional. Pero desde luego los porcentajes cortespondientes a FP y BUP son claramente significativos.

Tampoco las cifras obtenidas en las cuestiones destinadas a revisar la asimilación del concepto de función ofrecen lugar a dudas. La enorme heterogeneidad de respuestas encontradas para la cuestión sexta ( Explica con tus palabras qué es una función matemática. Da algun ejemplo.n) nos impide ofrecer una perspectiva global de la misma, por lo que la rectificación de su redacción se ha considerado conveniente para futuras experiencias. Sin embargo, los porcentajes correspondientes a la cuestión 7 ( Un científico mide y anota las longitudes de una varilla estando ésta a $5^{\circ}, 10^{\circ}, 15^{\circ}$, $20^{\circ} \ldots 50^{\circ} \mathrm{C}$ de temperatura. ¿Está construyendo una función?»), nos hace ver (Tabla IV), que un $60 \%$ de los alumnos admiten la existencia de una relación funcional entre series numéricas cuando entre ambas subyace una dependencia fisica, pero a través de la cuestión 8 ( $U$ Un profesor de $2^{\circ}$ de EGB anota durante los nueve meses del curso la estatura de uno de sus alumnos y las calificaciones que ha obtenido mensualmente. ¿Está construyendo una función?"), se puede observar que el peso porcentual disminuye (Tabla IV) significativamente al perderse dicha dependencia fisica, dándose además dicho descenso bajo una tónica muy peculiar, pues mientras en $7^{\circ}$ de EGB la relación funcional es aceptada por un inesperado $80 \%$ (es posible que la aleatoriedad haya jugado un papel no desdeñable), dicho nivel porcentual baja al $58,5 \%$ en FP y a un $47,1 \%$ en BUP. De nuevo parece que el proceso histórico vuelve a ser reprodu- cido intuitivamente por los alumnos. El hecho de que bajen los porcentajes en los niveles posteriores quizá se deba a que al introducirse las funciones en los cursos finales de la EGB y no haber tenido aún ocasión el alumno de aplicarlas sistemáticamente a la descripción y caracterización de fenómenos físico-químicos, se da una tendencia a retener sólo el aspecto puramente formal del concepto de función. Vemos como el mismo se va perdiendo pese al rigorismo con que normalmente es tratado en los textos de BUP. Había entonces que replantear el enfoque bajo el que transmitimos la idea de función matemática a nuestros estudiantes.

2. Mecánica. La posible asimilación de los conceptos de velocidad y aceleración era analizada, respectivamente, mediante las cuestiones 4,5 y 6 de primera etapa ("Imagina dos vehículos que se mueven a través de los círculos indicados en la figura (ver figura en Anexo I). $\mathrm{Si}$ ambos parten si-

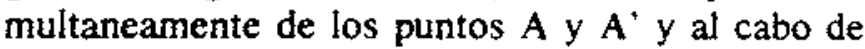
20 segundos se encuentran en $B^{\prime}$ y $B^{\prime}$ respectivamente, ¿cual llevará más velocidad, el que se mueve a los largo del círculo grande o el que lo hace a lo largo del pequeño?»: "Imagínate que para ir de Murcia a Madrid un coche tarda cinco horas y otro siete. ¿Cual de los dos va con más velocidad?"; "En el mismo tiempo que un coche va de Murcia a Cartagena otro va de Murcia a Alicante. La distancia de Murcia a Cartagena es de $50 \mathrm{~km}$ y la de Murcia a Alicante de 80 . ¿Cual de los dos coches irá con más velocidad") y 10 de la segunda ("Considera dos coches, uno de los cuales circula a 120 kilómetros por hora y el otro a $80 \mathrm{ki}$ lómetros por hora. ¿Cual de ellos tendrá mayor aceleración?"). A juzgar por las cifras obtenidas (Tabla V), el primero de estos conceptos (el de velocidad) es asimilado en un grado considerable (contestan correctamente a las tres cuestiones el $53,4 \%$ de los alumnos de los primeros niveles de EGB), pero es sumamente llamativo el altísimo porcentaje de alumnos de $7^{\circ}$ de EGB, $2^{\circ}$ de FP- 1 y $2^{\circ}$ de BUP que denotan no tener clara la idea de aceleración (imás del $70 \%$ en bachillerato!), to cual pone nítidamente de manifiesto, en concordancia con to enormemente dilatado y costoso de su génesis histórica, que es un concepto bastante más dificil de captar que lo que dan por supuesto, en forma implícita, la inmensa mayoría de los textos escolares, los cuales tienden a despacharlo con unas pocas líneas de caracter puramente teórico y bajo una perspectiva dogmático-evidente. Un enfoque crítico-histórico y una buena planificación de 
experiencias de laboratorio sería nuestra alternativa a esta cuestión.

Otra de las cuestiones normalmente tratadas con igual ligereza por los libros de texto es la relación matemática existente entre velocidad, espacio y tiempo en el movimiento unifomemente acelerado. Las preguntas 11 y 13 de la encuesta de segunda etapa ( Si dejamos caer simultáneamente dos objetos idénticos, uno desde una altura de 4 $\mathrm{m}$, y otro desde una altura de $8 \mathrm{~m} .:$ a) iel que cae desde los $8 \mathrm{~m}$ tardará el doble de tiempo en llegar al suelo que el que cae desde los 4 ? b) ilos tiempos de caida de cada objeto serán directamente proporcionales a las respectivas alturas?", "Imagina que un mismo objeto se deja caer primeramente desde una ventana situada a $10 \mathrm{~m}$ de altura y después de otra a $20 \mathrm{~m}$ de altura.: a) (las velocidades que tiene el objeto en el momento de llegar al suelo serán directamente proporcionales a las alturas desde las que cae? b) ¿Tendrá un valor doble la velocidad del objeto cuando cae desde la altura de $20 \mathrm{~m}$ que al caer desde la de $10 \mathrm{~m}$ ?") arrojan luz sobre esta cuestión, haciéndonos ver que el porcentaje de respuestas contradictorias, tanto en una como en otra cuestión, es bastante significativo (Tabla VI), apreciándose un notable descenso en FP (sobre el que no se nos ocurre explicación plausible) y siendo especialmente llamativo el valor observado en BUP (un $45 \%$ ), donde en buena lógica esta cuestión debía estar plenamente dominada. Como complemento al objetivo fundamental de estas preguntas recontamos por separado el porcentaje de alumnos que coincidian en contestar correctamente a los apartados "a» de ambas preguntas, intentando así ver si se tenía auténticamente claro el concepto de proporcionalidad directa. El máximo valor alcanzado por dicho porcentaje fue de $3,3 \%$ en el BUP, hablando la cifra por sí sola.

Con objeto de contrastar la persistencia en los estudiantes de la idea aristotélica sobre la necesidad de aplicar un motor a todo cuerpo en movimiento frente a la idea de acción a distancia, planteamos las cuestiones 14 y 15 en la segunda etapa (“iPuede moverse un cuerpo con velocidad constante sin que ningún otro objeto le comunique constantemente una fuerza que le mantenga la velocidad?», "Para que se mueva un objeto material ies imprescindible que algo o alguien le arrastre o empuje?"), encontrándonos con unos porcentajes de respuestas correctas verdaderamente reducidos (Tabla VII), puesto que en el mejor de los casos no se alcanza el diez por ciento.
En cuanto a la aceptación intuitiva por parte de los estudiantes de la composición de movimientos, los resultados que ofrece la cuestión 12 de segunda etapa ( Imagina que un joven que viaja a bordo de un transatlántico que marcha con una velocidad constante de $30 \mathrm{~km} / \mathrm{h}$ lanza verticalmente hacia arriba una pelota. ¿Donde caerá la pelota al volver hacia abajo, delante, detrás o encima del muchacho?»), no deja lugar a dudas (Tabla VIII): tan solo la tercera parte acepta dicha composición de movimientos en el mejor de los casos.

Las cuestiones 7 de $1^{a}$ etapa y 16 de $2^{a}$ ("Cuando un cuerpo se mueve con mayor velocidad, itiene más fuerza?"), como señalamos anteriormente, fueron planteadas para ver si el concepto de fuerza se asimilaba intuitivamente al de velocidad en lugar de al de aceleración. Los resultados obtenidos (Tabla IX) nos hacia ver que así era, y no se apreciaba una mejora sustancial al referirse al nivel de BUP.

El estudio en torno a la aceptación de la existencia del vacío y la posibilidad de realizarse en él fenómenos físicos, desarollado a través de las preguntas 17,19 y 21 de segunda etapa ("iPodria un cuerpo moverse en el vacío? ¿por qué?", "Se puede conseguir realmente el vacio en un laboratorio?" y "Pesan los cuerpos en el vacio ¿Por qué?»), nos indica en términos generales (Tabla X) una cierta resistencia a la idea de vacio, aunque dicha resistencia varía sensiblemente según el aspecto que se plantea.

Así más de la mitad estaba dispuesto a aceptar la posibilidad de moverse en el vacío, pero menos de un $20 \%$ aceptaban la posibilidad de que los cuerpos pesaran si habia vacio. Nos parece que las ideas de nuestros estudiantes al respecto están más influenciadas por las imágenes televisivas de los astronáutas flotando en el seno de las cápsulas espaciales ("moviéndose sin pesar») que por sus conocimientos académicos. los cuales se muestran insuficientes para hacerles superar sus ideas intuitivas al respecto.

Acabaremos la parte dedicada a Mecánica revisando, mediante los porcentajes de contestaciones correctas a las preguntas 20 y 23 de la encuesta de segunda etapa ( ¿Existe atracción gravitatoria entre todos los objetos imaginables (por ejemplo entre una silla y una mesa)?», " ¿Cuando será mayor la fuerza de atracción gravitatoria entre la Tierra y la Luna, cuando hay Luna llena o cuando la luna está en cuarto creciente?») el nivel de asimilación del concepto de gravedad (ver tabla XI). Es signi- 
ficativo el número de alumnos que generalizan el concepto de gravedad a todos los cuerpos, sobre todo en los niveles superiores (más del $65 \%$ ), pero a la hora de manejar operativamente tal concepto la baja en los porcentajes es uniforme y sensible.

3. Astronomía. Tal y como indicábamos anteriormente, las preguntas $22,27,28,29$ y 30 de la encuesta de segunda etapa, así como las $10,11,13$, 14 y 15 de la de primera, tenían como principal objetivo el darnos una información aproximada sobre el nivel general de conocimientos que tienen nuestros alumnos relativos a Astronomía. Dado que este objetivo se distancia un tanto del que hemos fijado como esencial para el presente trabajo tla persistencia intuitiva de errores similares a los cometidos históricamente), no nos parece procedente traer aquí la profija cantidad de cifras correspondientes a estas preguntas, pero sí que podemos afirmar a tenor de las mismas, que los conocimientos astronómicos de los alumnos era verdaderamente precarios, sin que se apreciara ningún progreso mínimamente sustancial al adentrarnos en los niveles de BUP y FP, situación ésta, a nuestro juicio, auténticamente lamentable.

La única cuestión de entre las que hemos estudiado en Astronomía, que entraba de lleno en el objetivo del presente trabajo, era la comparación de los mundos sublunar y supralunar. Sobre ello son bien ilustrativos los porcentajes obtenidos, conjuntamente, para las preguntas 12 de primera etapa y 24 y 25 de segunda. ("En la tierra como sabes hay hierro, oro, cartón, plomo, etc. ¿Habrá alguna de estas sustancias en Marte?», "¿Hay elementos químicos comunes en la Tierra y en otros planetas?", y "¿Hay elementos químicos comunes en la Tierra y en las estrellas?"). Los alumnos parecen establecer un aito grado de similitud entre la Tierra y los planetas (Tabla XII), adoptando tal posición más del $70 \%$ a partir de $7^{\circ}$ de EGB, pero no admiten tal similitud al efectuar la comparación con el mundo estelar, bajando entonces los porcentajes sensiblemente (oscilan entre el 30 y el $60 \%$ ), apreciándose por tanto una diferenciación entre el sistema solar y el universo situado fuera de sus límites. Aristóteles parece, pues, revivir en la mente de nuestros alumnos, que sólo se limitan a situar más lejana la frontera entre los dos mundos por él establecidos hace ya algunos siglos.

\section{CONCLUSION GENERAL}

A lo largo del análisis de los tres temas generales de estudio que hemos elegido, creemos haber aportado datos más que suficientes para apreciar la persistencia de un buen número de ideas intuitivas en nuestros estudiantes, tendentes a reproducir concepciones erróneas adoptadas en periodos históricos anteriores y que no han sido superadas a través de los conocimientos que se les imparten academicamente. Nuestra hipótesis de partida se confirma entonces, pero junto a las limitaciones que ya aceptábamos de entrada y que actuaban como acotaciones a la validez de nuestro estudio, hemos ido tomando paulatina conciencia de otras según ibamos desartollando el mismo. Desde la conveniencia de cambiar la redacción de algunas cuestiones a la palpable necesidad de anular series completas de las mismas, pasando por la revisión de nuestros métodos de tabulación.

Todo ello nos hace ver que no estamos sino al principio de una investigación, que dejamos abierta a otros equipos integrados en el tema, en la cual sólo hemos puesto los cimientos, pero que ya apunta hacia la necesidad imperiosa de proceder a un serio replanteamiento de nuestros programas y métodos de enseñanza de las ciencias, dentro del cual la utilización de un historicismo crítico tendría un relevante papel.

\section{REFERENCIAS BIBLIOGRAFICAS}

BRUSH, S.G., y KNNG, A.L. (eds), 1972, Hystory in the Teaching of Physics, (Proceeding of the Role of the Hystory of Physics in Physics Education) (University Press of New England: Hanover, New Hampshire).

NAVARRO, V., (ed), 1980, Actas del Simposio «La Historia de la Ciencia y la Enseñanzan (ICE Universidad de Valencia: Valencia).
PIAGET, J., 1969, Biologio y conocimiento (Siglo XXI: Madrid).

PIAGET, J., 1978. La representación del mundo en el niño (Morata: Madrid).

PIAGET, I.. 1979, Introducción a la epistemologia gentética (Paidos: Buenos Aires). 


\section{ANEXO I. ENCUESTA DE PRIMERA ETAPA}

1. Representa los quince primeros números por símbolos distintos a los que manejas habitualmente. Por ejemplo, $5=\square, 6=\mathbb{\Psi}, 7=\boldsymbol{\square}$.

2. Si en una caja tuvieses todos los números y en otra todos los números que empiezan por uno, ¿cual de las dos cajas crees que tendría más números?

3. Por una carretera pasan 100 coches al día $y$ por otra 1.000 ¿En cual de las dos carreteras habrá más accidentes? ¿Por qué?

4. Imagina dos vehículos que se mueven a través de los círculos indicados en la figura. Si ambos parten simultáneamente de los puntos $\mathrm{A}$ y $\mathrm{A}^{\prime}$ y al cabo de 20 segundos se encuentran en $B$ y $B^{\prime}$ respectivamente, ¿cual llevará más velocidad, el que se mueve a lo largo del círculo grande o el que to hace a lo largo del pequeño.

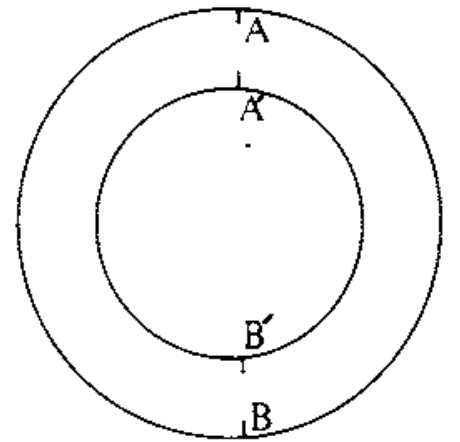

5. Imaginate que para ir de Murcia a Madrid un coche tarda 5 horas y otro 7. ¿Cual de los dos va con más velocidad?

6. En el mismo tiempo que un coche va de Murcia a Cartagena otro va de Murcia a Alicante. La distancia de Murcia a Cartagena es de 50 $\mathrm{km}$ y la de Murcia a Alicante de 80. ¿Cual de los dos coches irá con más velocidad?

7. Cuando un cuerpo va con más velocidad, ¿tiene más fuerza?

8. ¿Sabes la diferencia que hay entre los globos que si los sueitas suben al cielo y los que al soltarlos se caen al suelo?

9. ¿Tiene peso el aire?

10. ¿Donde está el sol cuando es de noche?

11. ¿Por qué cambia la forma de la luna?

12. En la tierra como sabes hay hierro, oro, carbón, plomo, etc. ¿Habrá alguna de estas sustancias en Marte?

13. ¿En qué se diferencia un planeta como Venus de una estrella?

14. ¿Tienen puntas las estrellas del cielo?

15. ¿Cómo se podría saber que la Tierra se mueve?

\section{ANEXO II. ENCUESTA DE SEGUNDA ETAPA}

1. Representa los quince primeros números por simbolos distintos a los que manejas habitualmente. Por ejemplo $5=\square, 6=\bigoplus, 7=\mathbb{~}$.

2. Sabiendo que $\sqrt{3}=1,7320 \ldots$ (infinitas cifras decimales) y que $3 / 4=0,75$ :

a) ¿Podria entonces construirse una varilla que midiese exactamente $3 / 4 \mathrm{~cm}$.?

b) ¿Y una que midiese exactamente $\sqrt{3} \mathrm{~cm}$.?

3. ¿Podría ocurrir que al medir alguna magnitud (masa, tiempo, área, volumen, etc) diera como resultado $\sqrt{-65}$ ?

4. ¿Qué hay más números naturales o números impares?

5. ¿Cual de los dos segmentos indicados en la figura tiene más puntos, el $A B$ o el CD? Razona la respuesta.

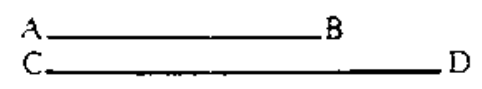

6. Explica con tus palabras qué es una función matemática. Da algún ejemplo.

7. Un cientifico mide y anota las longitudes de una varilla estando ésta a $5^{\circ}, 10^{\circ}, 15^{\circ}, 20^{\circ} \ldots$ $50^{\circ} \mathrm{C}$ de temperatura. ¿Está construyendo una función?

8. Un profesor de $2^{\circ}$ de EGB anota durante los nueve meses del curso la estatura de uno de sus alumnos y las calificaciones que ha obtenido mensualmente. ¿Está construyendo una función?

9. ¿Podría suceder que la suma de infinitos números (del tipo que sean) no dé como resultado infinito?

10. Considera dos coches, uno de los cuales circula a $120 \mathrm{~km} / \mathrm{h}$ y el otro a $80 \mathrm{~km} / \mathrm{h}$ ¿Cual de eilos tendrá mayor aceleración? ¿Por qué?

11. Si dejamos caer simultáneamente dos objetos idénticos, uno desde una altura de 4 metros y otro desde una altura de 8 metros:

a) ¿el que cae desde los 8 metros tardará el doble de tiempo en llegar al suelo que el que cae desde los 4 ?

b) ¿los tiempos de caida de cada objeto serán directamente proporcionales a las respectivas alturas?

12. Imagina que un joven que viaja a bordo de un transatlántico que marcha con una velocidad constante de $30 \mathrm{~km} / \mathrm{h}$ lanza verticalmente hacia arriba una pelota. ¿Donde caerá la pelota al volver hacia abajo, delante, detrás o encima del muchacho? 
13. Imagina que un mismo objeto se deja caer primeramente desde una ventana situada a $10 \mathrm{~m}$. de altura y después de otra a $20 \mathrm{~m}$. de altura: a) ¿las velocidades que tiene el objeto en el momento de llegar al suelo serán directamente proporcionales a las alturas desde las que cae? b) ¿Tendrá un valor dobie la velocidad del objeto cuando cae desde la altura de $20 \mathrm{~m}$. que al caer desde la de 10 ?

14. ¿Puede moverse un cuerpo con velocidad constante sin que ningún otro objeto le comunique constantemente una fuerza que le mantenga la velocidad?

15. Para que se mueva un objeto material ies imprescindible que algo 0 alguien lo arrastre $o$ empuje?

16. Cuando un cuerpo se mueve con mayor velocidad. ¿Tiene más fuerza?

17. ¿Podría un cuerpo moverse en el vacío? ¿Por qué?

18. Si lanzas un cuerpo verticalmente hacia arriba, ¿cuando irá con mayor velocidad, en el momento del lanzamiento, en el punto más alto de la trayectoria o en el momento de llegar al suelo? Razona la respuesta.

19. ¿Se puede conseguir realmente el vacio en un laboratorio?
20. ¿Existe atracción gravitatoria entre todos los objetos imaginables (por ejemplo entre una silla y una mesa)?

21. ¿Pesan los cuerpos en el vacio? ¿Por qué?

22. ¿Por qué cambia la forma de la luna?

23. ¿Cuando será mayor la fuerza de atracción gravitatoria entre la Tierra y la Luna, cuando hay Luna liena o cuando la Luna está en cuarto creciente?

24. ¿Hay elementos químicos comunes en la Tierra y en otros planetas?

25. ¿Hay elementos químicos comunes en la Tierra y en las estrellas?

26. ¿Cual es la diferencia entre los planetas y las estrellas?

27. ¿Como se puede distinguir a simple vista los planetas de las estrellas?

28. ¿Están todas las estrellas a la misma distancia de la Tierra?

29. ¿Aparecen todas las noches en el cielo las mismas estrellas?

30. ¿Por qué se suceden a lo largo del año diferentes estaciones climáticas?

TABLA I

UTILIZACION DEL CERO Y OEL PRINCIPIO DEL VALOR RELATIVO

Porcentajes de respuestas positivas a la cuestión l de ambas

encuestas.

\begin{tabular}{lcc}
$\frac{\text { CURSO }}{3^{\circ} \mathrm{EGB}}$ & UTILIZACION OEL & UTILIZAN VALOR RELATIVO \\
\cline { 2 - 3 } $5^{\circ} \mathrm{EGB}$ & 5,1 & 0,0 \\
$7^{\circ} \mathrm{EGB}$ & 3,1 & 0,0 \\
$2^{\circ} \mathrm{FP}-1$ & 0,0 & 2,5 \\
$2^{\circ} \mathrm{BUP}$ & 3,2 & 8,5 \\
& 4,1 & 14,9
\end{tabular}


IABLA II CONCEPTO Y MANIPULACION OE LOS INFINITOS

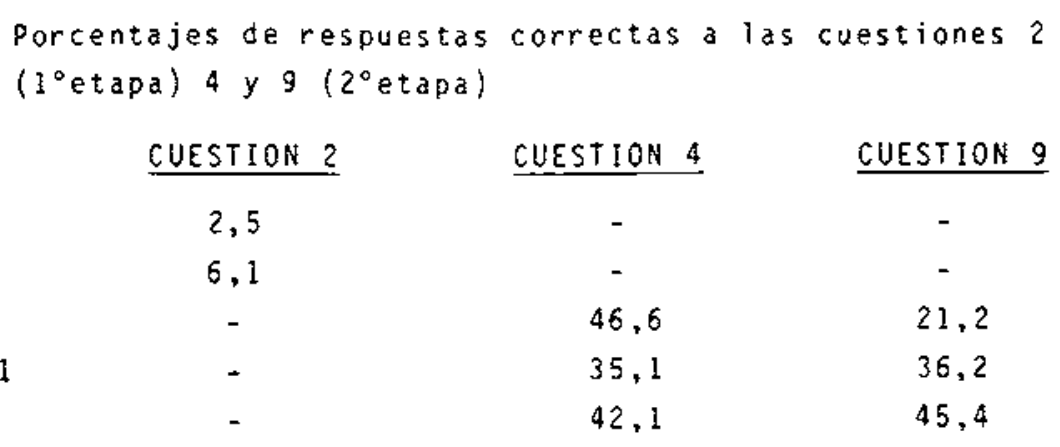

TABLA III NUMEROS IRRACIONALES

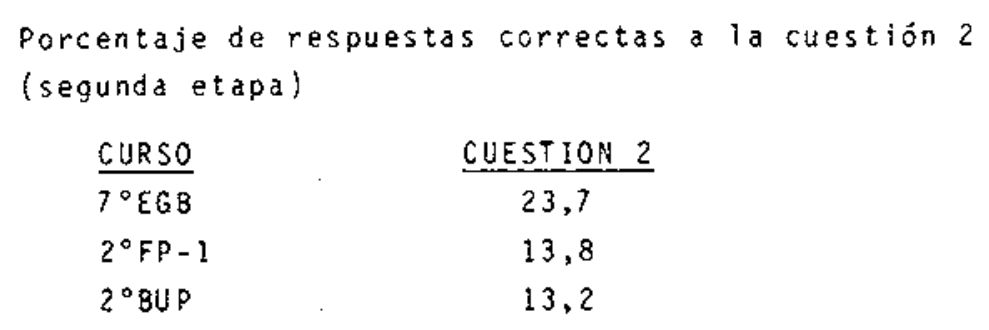

TABLA IV CONCEPTO DE FUNCION

\begin{tabular}{|c|c|c|}
\hline CUR50 & CUESTION 7 & CUESTION 8 \\
\hline $7^{\circ} \mathrm{EGB}$ & 64,4 & 80,5 \\
\hline $2^{\circ} 89-1$ & 59,5 & 58,5 \\
\hline $2^{\circ} 8 \cup P$ & $7 i, 9$ & 47,1 \\
\hline
\end{tabular}

IABLA V CONCEPTO DE ACELERACION

Porcentaje de respuestas correctas a la cuestión 10
(encuesta $2^{\circ}$ etapa)
$\begin{array}{ll}\text { CURSO } & \frac{\text { CUESTION }}{5,1} \\ \text { FPEGB } & 19,1 \\ \text { BUP } & 28,9\end{array}$

TA8LA VI

RELACION ENTRE VELOCIOAD, ESPACIO Y TIEMPO EN EL MOVIMIENTO UNIFORMEMENTE ACELERADO.

Porcentaje de respuestas contradictorias observadas en las cuestiones 11 y 13 (encuesta $2^{\circ}$ etapa)

\begin{tabular}{lcc} 
CURSO & CUESTION 11 & CUESTION I3 \\
\hline $7^{\circ}$ EGB & 34,7 & 50,8 \\
FP & 24,4 & 29,8 \\
BUP & 44,6 & 44,6
\end{tabular}


TABLA VII

IDEA DE ACCION A DISTANCIA

Dorcentaje de respuestas correctas observadas en las cuestiones

14 y 15 (encuesta $2^{\circ}$ etaod). considersolas conjuntamente

$\begin{array}{lc}\text { CURSO } & \text { CUESTION } 14-15 \\ 7=G G E & 6,8 \\ \text { FP } & 5,3 \\ \text { BUP } & 9,9\end{array}$

$\underline{T B B A P Y I I}$

COMPOS IC ION DE MOVIMIEN TOS

Porcentaje de respuestas correctas a la cuestión 12 (encuesta de segunda etapa).

\begin{tabular}{lc} 
CLIRSO & CLESTION 12 \\
\hline$T^{\circ} E G B$ & 11.0 \\
FE & 33,0 \\
$B$ B P & 33,0
\end{tabular}

TABLA IX

ASIMILACION DE FUERZA CON YELOCIDAD

Porcentaje de resouestas correctas a las cuestiones 7 fencuesta primera etapa) y 16 (encuesta segunda etapa\}.

\begin{tabular}{lcc} 
CURSO & CUESTION 7 & CUESTION 16 \\
\hline $3^{\circ} \mathrm{EGB}$ & 5,9 & - \\
$5^{\circ} \mathrm{EGB}$ & 18,5 & - \\
$7^{\circ} \mathrm{EGB}$ & - & 16,7 \\
FP & - & 31,9 \\
BUP & - & 22,3
\end{tabular}

IABLA $X$

ACEPTACION OE LA IDEA DE VACIO

Porcentaje de respuestas afirmativas a las cuestiones 17,19 y 21 (encuesta segunda etapa).

\begin{tabular}{|c|c|c|c|}
\hline CURSO & CUESTION 17 & CUEST ION 19 & CUESTION 21 \\
\hline $7^{\circ} E G B$ & 57,6 & 53,2 & 54,5 \\
\hline$F P$ & 44,1 & 44,7 & 39,7 \\
\hline BUP & 15,3 & 20,2 & 21,5 \\
\hline
\end{tabular}

TABLA XI

ATRACCION GRAVITATORLA

Porcentaje de respuestas afirmativas a las cuestiones 20 y 23 (Encuesta segunda etapa)

\begin{tabular}{lcc} 
CURSO & CUESTION 20 & CUESTION 23 \\
\hline $7^{\circ}$ EGB & 55,1 & 28,0 \\
FP & 78,7 & 61,7 \\
BUP & 66,1 & 47,9
\end{tabular}

\section{TA8LA XII}

COMPARACION MUNDOS SUBLUNAR Y SUPRAL UNAR

Porcentaje de respuestas afirmativas a las cuestiones 12 (encuesta primera etapa), 24 y 25 (encuesta segunda etaja)

\begin{tabular}{lccc} 
CURSO & CUESTION 12 & CUESTION 24 & CUESTION 25 \\
\cline { 2 - 3 } $3^{\circ} E G 8$ & 30,5 & - & - \\
$5^{\circ}$ EG8 & 40,8 & - & - \\
$7^{\circ}$ EGB & - & 72,0 & 41,1 \\
FP & - & 76,6 & 55,3 \\
BUP & - & 89,1 & 53.0
\end{tabular}

